\title{
26
}

\author{
DE \\ COLOPROCTOLOGIA
}

4

OUTUBRO / DEZEMBRO 2006

ARTIGOS ORIGINAIS

\section{Doenças Anais Concomitantes à Doença Hemorroidária: Revisão de 1.122 Pacientes}

\author{
Anal Diseases Associated to Hemorrhoids: \\ Review of 1.122 Patients
}

\author{
GERALDO MAGELAGOMES DACRUZ ${ }^{1}$; JORGELUIZSANTANA ${ }^{2}$; SANDRA KELY ALVES \\ DE ALMEIDA SANTANA ${ }^{2}$; RENATAMAGALI RIBEIRO SILLUZIO FERREIRA ${ }^{3}$; PETERSON MARTINS NEVES $^{3}$; \\ MARINA NEVES ZERBINI DEFARIA ${ }^{4}$
}

(1) Mestre, Doutor e Professor Titular de Coloproctologia, TSBCP, TFBG, TALACP, TCBC, TISUCRS, FASCRS;

(2) Pós-graduandos lato sensu em Coloproctologia; (3) Assistentes do Grupo de Coloproctologia; (4) Estagiária Voluntária do Serviço de Coloproctologia

CRUZ GMG; SANTANA JL; SANTANA SKAA; FERREIRA RMRS; NEVES PM; FARIA MNZ. Doenças Anais Concomitantes à Doença Hemorroidária: revisão de 1.122 Pacientes. Rev bras Coloproct, 2006;26(3): 369-376.

RESUMO: Em 34.000 pacientes coloproctológicos foi feito o diagnóstico de doença hemorroidária (DH), como doença coloproctológica principal, em 9.289 pacientes $(27,3 \%)$, dos quais $1.122(12,1 \%)$ eram portadores de doenças anais concomitantes à DH (DAC). Dos 9.289 portadores de DH, 2.417 foram operados de DH (26,0\%) e destes, 729 foram operados, ao mesmo tempo, de DAC (30,2\%). Assim, dos 1.122 portadores de DAC, 729 foram operados delas $(65,0 \%)$. Em relação aos 9.289 portadores de DH, a DAC mais comum foi a fissura anal (541 casos, 5,8\%), seguida de hipertrofia de papilas anais (312 casos, 3,4\%), fístulas anais (117 casos, $1,3 \%)$, hipotonia anal com incontinência parcial (112 casos, $1,2 \%)$, condilomas anais acuminados $(37$ casos, $0,4 \%)$ e tumores anais $(3$ casos, $0,03 \%)$; e a mesma ordem foi verificada em relação às 1.122 DAC: fissura anal $(48,2 \%)$, hipertrofia de papilas anais $(\mathbf{2 7 , 8 \%})$, fístulas anais $(\mathbf{1 0 , 4 \%})$, hipotonia anal com incontinência parcial $(\mathbf{1 0 , 0 \%})$, condilomas anais acuminados $(3,3 \%)$ e tumores anais $(0,3 \%)$. Em relação à cirurgia, das 1.122 DAC 729 foram operadas $(65,0 \%)$ nesta ordem: fissura anal $(317$ casos, $28,3 \%)$, hipertrofia de papilas anais ( 267 casos, $23,8 \%)$, fístulas anais (89 casos, $7,9 \%)$, hipotonia anal com incontinência parcial (31 casos, $\mathbf{2 , 8 \%}$ ), condilomas anais acuminados (22 casos, $1,9 \%)$ e tumores anais ( 3 casos $0,3 \%)$; e em relação às próprias DAC as incidências de cirurgias foram: tumor anal $(\mathbf{1 0 0 , 0} \%)$, hipertrofia de papilas anais $(\mathbf{8 5 , 6 \%})$, fístulas anais $(\mathbf{7 6 , 0} \%)$, condilomas anais acuminados

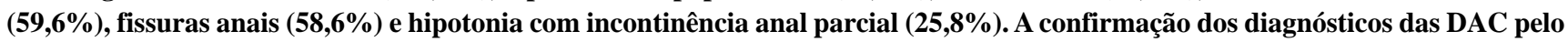
exame histopatológico foi de $\mathbf{7 2 , 8 \%}$, em ordem decrescente: condilomas anais e fístulas anais $(\mathbf{1 0 0 , 0} \%)$, hipertrofia de papilas anais $(79,0 \%)$, fissuras anais $(68,5 \%)$ e tumores anais $(66,7 \%)$.

Descritores: Doenças anais e hemorróidas.

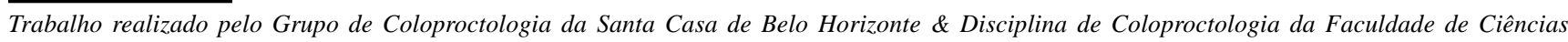
Médicas de Minas Gerais.

$\overline{\text { Recebido em 26/06/2006 }}$

Aceito para publicação em 14/08/2006 


\section{INTRODUÇÃO}

As hemorróidas são verdadeiros coxins de tecido conjuntivo fibroelástico, ricos em plexos vasculares e com múltiplas anastomoses arteriovenosas, situados na submucosa da região anorretal, que se comportam como uma "almofada", ajudando na continência anal, permitindo a chamada "oclusão anal de repouso" 1, 2, 8, 9, 11, 12, 13, 26, 28, 29, 30, 33 . Do ponto de vista etiopatogênico, o reconhecimento anatômico destes coxins reforça a teoria do "deslizamento do assoalho anal" proposta por Haas et al. ${ }^{19}$, que explica a evolução das hemorróidas ao longo das várias faixas etárias, com aparecimento dos sintomas em virtude da perda de elasticidade e da ruptura dos elementos estruturais de sustentação, devido aos fatores involutivos naturais que, sob a ação dos fatores desencadeantes de esforço, facultariam o referido deslizamento. Devem ser salientados, além destes fatores de sustentação, os fatores vasculares e a micro-circulação dos plexos hemorroidários, além da elevação da pressão máxima de repouso ao nível do canal anal associada à presença de hemorróidas, enfatizada por Santos ${ }^{30}$.

Quanto à localização anatômica, a DH pode ser classificada em "internas" (submucosa anorretal acima do ligamento de Parks ${ }^{27}$, recobertas por epitélio mucoso), "externas" (abaixo do ligamento de Parks, recobertas por pele modificada do canal anal) e "mistas" (extensões internas e externas). Quanto à disposição em torno do canal anal - uma vez que os dos vasos hemorroidários superiores seguem uma distribuição anatômica bem definida na grande maioria das pessoas, formando três "coxins" ou "mamilos" mais volumosos ou principais ou primários, situados na submucosa anorretal - os mamilos ou coxins distribuem-se nas posições lateral esquerda, ântero-lateral direita e póstero-lateral direita (duas, cinco e nove horas, em analogia aos ponteiros do relógio afixado no períneo do paciente em posição genupeitoral) ${ }^{8,9}$, 13, 26, 28, 29, 30, 33 .

A região perianal, o ânus, o canal anal e o reto podem ser sedes de um sem número de doenças, devendo estas regiões anatômicas ser examinadas com detalhes, mesmo tendo sido encontrada a doença mais comum ai existente: a doença hemorroidária ${ }^{3,5,6,8,9,15}$, $16,17,18,22,23,24,27,30,31,32,34$. Isto, por que várias doenças podem sediar tais regiões além da $\mathrm{DH}$, somando seus sintomas aos desta doença.
Doenças proctológicas que convivem com a DH podem ser de várias naturezas, constituindo as denominadas doenças anais concomitantes (DAC) à DH. Elas podem ser classificadas de várias formas, sendo uma boa classificação aquela que as diferencia pelo próprio exame proctológico. Assim, podem ser divididas em (a) doenças estáticas encontradas pelo exame proctológico e (b) doenças encontradas à inspecção e por manobras de esforço e que se exteriorizam pelo ânus $8,9,11,12,26,30$.

Dentre as primeiras, as doenças encontradas pelo exame proctológico (inspecção, toque retal e anuscopia) podem ser citadas as seguintes DAC: (1) lesões cutâneas ${ }^{28}$ (câncer de pele perianal $7,8,9,10,11,13$, 22, 27, 28, 32, condilomas 3, 5, 7, 8, 9, 22, 28, 30, úlceras 7, 8, 9, 28,

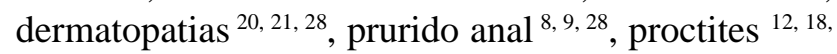
${ }^{22,30}$ e destruição anal e perianal ${ }^{8,9,11}$ ); (2) lesões da mucosa do canal anal (fissura 15, 18, 22, 27, 30, hipertrofia de papilas anais ${ }^{15,18,22,27,30}$, criptite ${ }^{15,18,22,27,30}$, proctite 15, 18, 22, 27, 30, câncer transicional 11, 13, 27, 22, 27, 32); (3) tumores duros subcutâneos (gânglios, nódulos fibróticos, granulomas, hematomas, massas tumorais benignas e malignas) $11,13,27,22,27,32$; (4) tumores macios subcutâneos (abscessos, cistos, lipomas, hematomas) 11, 13, 27, 22, 27, 32; (5) infiltrações enduradas fazendo corpo com a pele (doença de Crohn, hidroadenite supurativa, infiltração carcinomatosa) 6, 8, 9, 10, 24, 27,30; (6) lesões anorretais inespecíficas (doença de CROHN, RCUI) $6,8,9,10,24,27,30$; (7) lesões anorretais específicas (tuberculose $20,21,27,28$, esquistossomose $20,21,27,31$, actino e blastomicose ${ }^{16}$, amebíase ${ }^{20,21,27,31}$ ); (8) estenoses anais (por fissuras, cirúrgicas, carcinomatosas) ${ }^{11,13 \text {, }}$ 22, 27, 32; (9) orifícios e trajetos fistulosos (fístulas perianais e anorretais, específicas e inespecíficas) ${ }^{15}$, 17, 22, 27, 30; (10) anormalidades com o tônus anal (hipertonia, hipotonia, atonia) ${ }^{8,9,17,20,21,26,28,30}$; (11) invasão do ânus e períneo por doenças que vêm do reto (tumores retais); (12) comprometimento anal de doenças sistêmicas (diabete, pênfigo, linfoma) ${ }^{8,9,17}$, 20, 21, 26, 28, 30; e (13) doenças dermatológicas que podem comprometer as regiões anal e perianal e doenças intestinais que podem apresentar lesões cutâneas próximas dessas regiões ou à distância (psoríase com suas lesões mais eritematosas que escamosas quando na região perianal e o eritema nodoso, frequentemente em localização pré-tibial, que pode acompanhar a retocolite ulcerativa) ${ }^{20,21,28}$.

Dentre as doenças encontradas à inspecção e por manobras apalpatórias e de esforço e que se 
Tabela 1 - Incidência de pacientes portadores de doença hemorroidária, de portadores de doenças anais concomitantes e de pacientes operados.

\begin{tabular}{lrc}
\hline Doença Hemorroidária \& DAC & $\mathbf{N}$ & $\mathbf{\%}$ \\
\hline N pacientes atendidos & 34.000 & $27,3 \%$ \\
N portadores de DH & 9.289 & $12,1 \%$ \\
N DAC & 1.122 & $26,0 \%$ \\
& & $30,2 \%$ \\
N pacientes operados de DH & 2.417 & $65,0 \%$ \\
N operados de DAC : N operados de DH & $729: 2.417$ & \\
N operados de DAC : N portadores de DAC & $729: 1.122$ & \\
\hline
\end{tabular}

exteriorizam pelo ânus podem ser consideradas: hemorróidas internas, prolapsos e procidências retais, pólipos, papilas anais hipertrofiadas, tumores vilosos prolapsados 7, 8, 9, 10, 12, 15, 17, 18, 22, 24, 27, 30.

É de fundamental importância a identificação de tais ocorrências (doenças anais concomitantes DAC), tanto por que podem ser doenças a serem abordadas cirurgicamente antes da DH (e.g., câncer, doença de Crohn) $7,8,9,10,12,15,17,18,22,24,27,30$, como doenças que devem ser abordadas clinicamente antes da abordagem cirúrgica da DH (e.g., proctites específicas e não específicas) ${ }^{8,9,17,20,21,26,28,30}$ e doenças que podem ser abordadas em conjunto com a hemorroidectomia (e.g., fissuras anais, hipertrofia de papila anal, estenose anal, fístula anal, condiloma anal acuminado) 7, 8, 9, 10, 12, 15, 17, 18, 22, 24, 27, 30 .

\section{OBJETIVO}

O objetivo deste trabalho é estudar 1.122 pacientes que receberam diagnósticos de doenças anais concomitantes (DAC) à doença hemorroidária (DH), analisando dados epidemiológicos (idade e sexo), e operadas em concomitância com a DH.

\section{Casuística: pacientes $\&$ métodos}

Em 34.000 pacientes coloproctológicos foi feito o diagnóstico de $\mathrm{DH}$ como doença coloproctológica principal em 9.289 pacientes $(27,3 \%)$ e destes, 1.122 apresentavam várias doenças anais concomitantes (DAC) à $\mathrm{DH}(12,1 \%)$. Dos 9.289 pacientes portadores de DH, $2.417(26,0 \%)$ foram submetidos à hemorroidectomia, dentre os quais, 729 eram portadores de DAC. O material é constituído pelos 1.122 portadores e os 729 operados de DAC.

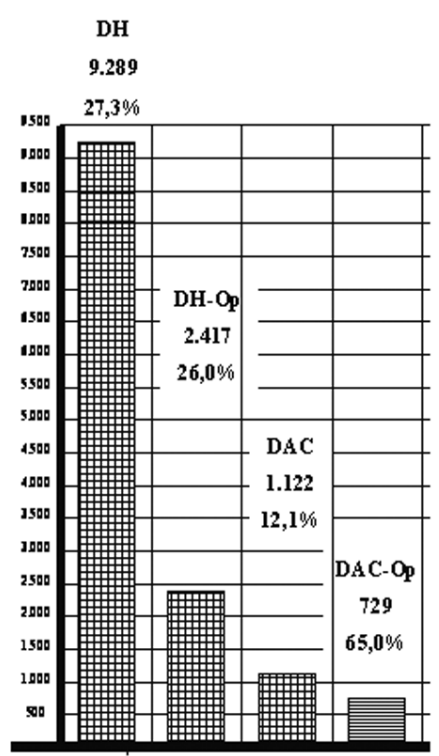

Figura 1 - Figura ilustrativa de pacientes portadores de doença hemorroidária, de doenças anais concomitantes e de pacientes operados.

\section{RESULTADOS}

Incidência absoluta e relativa de doenças anais concomitantes à doença hemorroidária, diagnosticadas e operadas simultaneamente: dos 34.000 pacientes atendidos 9.289 eram portadores de $\mathrm{DH}(27,3 \%), 1.122$ dos quais $(12,1 \%)$ receberam também diagnóstico de DAC; dos 9.289 portadores de $\mathrm{DH}$, 2.417 foram operados da $\mathrm{DH}(26,0 \%)$, entre os quais 729 portadores de DAC (30,2\%). Em relação aos 1.122 portadores de DAC à DH, os 729 operados representam 65,0\% (Tabela 1 e Figura 1).

Incidência das várias DAC concomitantes à $\mathrm{DH}$ em relação à mesma e em relação às $\mathrm{DAC}$ : dos 1.122 pacientes portadores de $\mathrm{DAC}$ à $\mathrm{DH}, 541$ 
Rev bras Coloproct

Outubro/Dezembro, 2006
Doenças Anais Concomitantes à Doença Hemorroidária: revisão de 1.122 pacientes

Geraldo Magela Gomes da Cruz e Cols.
Vol. 26 $\mathbf{N}^{\circ} 4$

Tabela 2 - Incidência de doenças anais concomitantes à DH (coluna A), de doenças anais concomitantes (coluna B) e entre as doenças anais concomitantes (coluna C).

\begin{tabular}{lccc}
\hline & A & B & C \\
\cline { 2 - 4 } DAC à DH & $\begin{array}{c}\text { N-DAC relativo } \\
\text { a 9.289 DH }\end{array}$ & $\begin{array}{c}\text { \%-DAC relativo } \\
\text { a 9.289 DH }\end{array}$ & $\begin{array}{c}\text { \%-DAC relativo } \\
\text { a 1.122 DAC }\end{array}$ \\
\hline Fissura anal & 541 & $5,8 \%$ & $48,2 \%$ \\
Hipertrofia de papilas anais & 312 & $3,4 \%$ & $27,8 \%$ \\
Fístula anal & 117 & $1,3 \%$ & $10,4 \%$ \\
Hipotonia com incont. parcial & 112 & $1,2 \%$ & $10,0 \%$ \\
Condilomas acuminados & 37 & $0,4 \%$ & $3,3 \%$ \\
Tumor perianal & 3 & $0,03 \%$ & $0,3 \%$ \\
Total & 1.122 & $12,1 \%$ & $100,0 \%$ \\
\hline
\end{tabular}

tinham fissura anal (5,8\%), 312 tinham hipertrofia de papilas anais $(3,4 \%), 117$ tinham fístula anal $(1,3 \%)$, 112 tinham hipotonia anal com incontinência fecal parcial $(1,2 \%), 37$ tinham condilomas anais acuminados $(0,4 \%)$ e 3 tinham tumores perianais $(0,03 \%)$ (colunas A e B da Tabela 2 e Figura 2). Os percentuais das várias $\mathrm{DAC}$ à $\mathrm{DH}$ em relação a elas próprias foram $48,2 \%$ de fissuras anais, $27,8 \%$ de hipertrofia de papilas anais, $10,4 \%$ de fístulas anais, $10,0 \%$ de hipotonia com incontinência fecal parcial, $3,3 \%$ de condilomas anais acuminados e $0,3 \%$ de tumores perianais) (coluna $\mathbf{C}$ da Tabela 2 e Figura 2).

Incidência de DAC à DH diagnosticadas e operadas em relação a elas próprias e a cada uma delas: 317 dos 541 casos de fissura anal foram operados $(58,6 \%)$, o que corresponde a $28,3 \%$ das 1.122 DAC diagnosticadas; 267 dos 312 casos de hipertrofia de papilas anais foram operados $(85,6 \%)$, o que corresponde a $23,8 \%$ das 1.122 DAC diagnosticadas; 89 dos 117 casos de fístulas anais foram operados (76,0\%), o que corresponde a 7,9\% das 1.122 DAC diagnosticadas; 31 dos 112 casos de hipotonia anal com incontinência fecal parcial foram operados $(25,8 \%)$, o que corresponde a $2,8 \%$ das 1.122 DAC diagnosticadas; 22 dos 37 casos de condilomas anais acuminados foram operados $(59,5 \%)$, o que corresponde a $1,9 \%$ das 1.122 DAC diagnosticadas; os 3 casos de tumor perianal foram operados $(100,0 \%)$, o que corresponde a $0,3 \%$ das 1.122 DAC diagnosticadas (Tabela 3 e Figura 3).

Incidência de DAC à $\mathrm{DH}$ diagnosticadas e operadas em relação a elas próprias e a cada uma delas: Dos 1.122 portadores de DAC, 729 foram operados, tendo o exame histopatológico corroborado

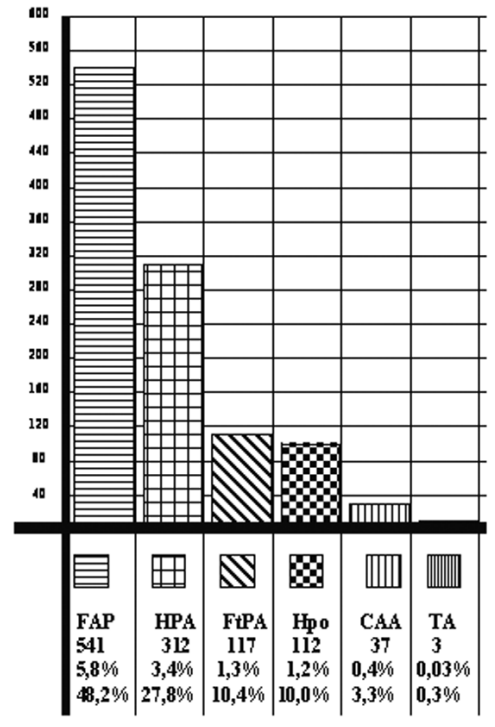

Figura 2 - Incidências de DAC diagnosticadas ao exame proctológico (1.122 casos) - fissura anal posterior (FAP), hipertrofia de papila anal (HPA), fistula perianal (FtPA), hipotonia esfincteriana (Hpo), condiloma anal acuminado (CAA) e tumor perianal (TPA) , em relação aos pacientes portadores de doença hemorroidária, em números absolutos e percentuais e em relação às próprias doenças anais concomitantes.

os diagnósticos proctológicos em 510 espécimes cirúrgicos $(72,8 \%)$, excluindo-se os 31 casos de hipotonia anal, não passíveis de diagnóstico histopatológico: dos 541 casos de fissura anal, 317 foram operadas e o exame histopatológico corroborou o diagnóstico em 217 espécimes cirúrgicos $(68,5 \%)$; dos 312 casos de hipertrofia de papilas anais, 267 foram operadas e o exame histopatológico corroborou o diagnóstico em 211 espécimes cirúrgicos (79,0\%); dos 117 casos de fístulas anais, 89 foram operados e em todos os casos o exame 
Tabela 3 - Incidências de doenças anais concomitantes - fissura anal posterior (FAP), hipertrofia de papila anal (HPA), fístula perianal (FtPA), hipotonia esfincteriana (Hpo), condiloma anal acuminado (CAA) e tumor perianal (TPA) - (coluna A), de doenças anais concomitantes operadas em números absolutos (coluna $B$ ) e percentuais (coluna $C$ ) e em ralação à cada doença anal concomitante (coluna D).

\begin{tabular}{lcccc}
\hline & A & B & C & D \\
\cline { 2 - 5 } Doenças Anais (DAC) à DH & N-DAC & N-DAC operada & $\begin{array}{c}\text { \%-DAC } \\
\text { operados:1.122 }\end{array}$ & $\begin{array}{r}\text { \%-DAC: N da } \\
\text { DAC específica }\end{array}$ \\
\hline Fissura anal & 541 & 317 & $28,3 \%$ & $58,6 \%$ \\
Hipertrofia de papilas anais & 312 & 267 & $23,8 \%$ & $85,6 \%$ \\
Fístula anal & 117 & 89 & $7,9 \%$ & $76,0 \%$ \\
Hipotonia com incont. parcial & 112 & 31 & $2,8 \%$ & $25,8 \%$ \\
Condilomas acuminados & 37 & 22 & $1,9 \%$ & $59,5 \%$ \\
Tumor perianal & 3 & 3 & $0,3 \%$ & $100,0 \%$ \\
Total & 1.122 & 729 & $65,0 \%$ & $65,0 \%$ \\
\hline
\end{tabular}

histopatológico corroborou o diagnóstico (100,0\%); os 31 casos operados dos 112 casos de hipotonia esfincteriana com incontinência anal parcial não são passíveis de diagnóstico histopatológico; dos 37 casos de condiloma acuminado, 22 foram operados e o exame histopatológico corroborou o diagnóstico em todos os 22 espécimes cirúrgicos $(100,0 \%)$; os 3 casos de tumor anal foram operados e o exame histopatológico corroborou o diagnóstico em 2 espécimes cirúrgicos $(66,7 \%)$ (Tabela 4 e Figura 4).

\section{DISCUSSÃO}

Incidência absoluta e relativa de doenças anais concomitantes à doença hemorroidária, diagnosticadas e operadas simultaneamente: há muita controvérsia sobre a epidemiologia da $\mathrm{DH}$, e trabalhos, os mais diversos, têm sido publicados com referência ao estudo da incidência de DH na população, e os dados têm sido conflitantes, com autores estabelecendo incidências médias que vão de próximos a 4,4\%, como Johanson et al ${ }^{23}$ a $86 \%$ da humanidade, conforme afirmado por Hass et al ${ }^{19}$; e passando por incidências em torno de $10 \%$, como admitem Ganchrow et al. ${ }^{14} \mathrm{e}$ Iwagaki et al. ${ }^{22}$. Isto faz concluir existência de metodologias completamente diversas, e que pode eivar de erros conclusões precipitadas, pois vão desde a denominação para a DH sintomática até achados de necrópsias. Em estudo comparativo entre grupos sintomáticos e assintomáticos, Hass et al. ${ }^{19}$ relatam uma incidência bastante semelhante, com identificação de hemorróidas em $88 \%$ no grupo sintomático (DH), e

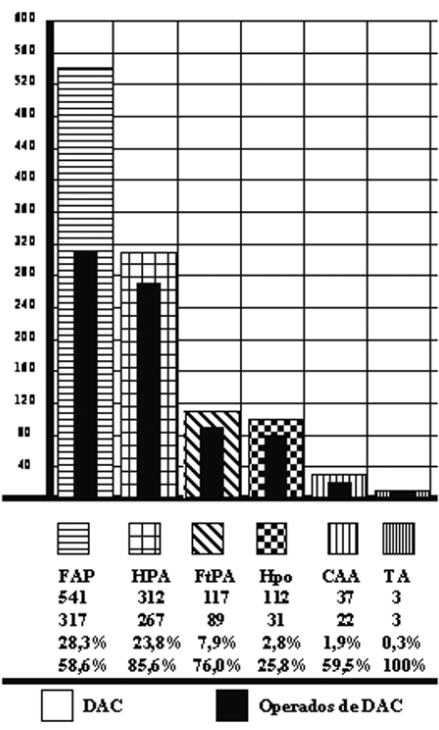

Figura 3 - Incidências de doenças anais concomitantes (DAC) diagnosticadas (1.122 casos) e incidência de DAC operadas (729 casos) - fissura anal posterior (FAP), hipertrofia de papila anal (HPA), fístula perianal (FtPA), hipotonia esfincteriana (Hpo), condiloma anal acuminado (CAA) e tumor perianal (TPA).

$82 \%$ no grupo assintomático (submetido a exame proctológico de rotina para rastreamento de câncer colorretal ou por solicitação de outras clínicas).

No decurso de 38 anos de profissão com prática totalmente centrada em Coloproctologia, tivemos a oportunidade de formar um fichário constituído de 34.000 pacientes, tendo feito diagnóstico de DH como doença de fundo e motivo da consulta, portanto sintomáticas, em 9.289 pacientes $(27,32 \%)$ e operado 2.417 
Rev bras Coloproct

Outubro/Dezembro, 2006
Doenças Anais Concomitantes à Doença Hemorroidária: revisão de 1.122 pacientes

Geraldo Magela Gomes da Cruz e Cols.
Vol. 26 $\mathbf{N}^{\circ} 4$

Tabela 4 - Incidência absoluta de doenças anais concomitantes (DAC) à doença hemorroidária (DH) (coluna A), de doenças anais concomitantes operadas (coluna B), de números absolutos (coluna $C$ ) $e$ percentuais (coluna D) de diagnósticos histopatológicos em espécimes cirúrgicos.

\begin{tabular}{|c|c|c|c|c|}
\hline & $\mathbf{A}$ & B & $\mathbf{C}$ & D \\
\hline $\begin{array}{l}\text { DAC operadas em } \\
\text { conjunto com a DH }\end{array}$ & $\mathrm{DAC}+\mathrm{DH}$ & $\begin{array}{c}\text { N operados } \\
\text { DAC }+ \text { DH }\end{array}$ & $\mathbf{N}$ diagn EHP & \% diagn EHP \\
\hline Fissura anal & 541 & 317 & 217 & $68,5 \%$ \\
\hline Hipertr. de papila anal & 312 & 267 & 211 & $79,0 \%$ \\
\hline Fístula anal & 117 & 89 & 89 & $100,0 \%$ \\
\hline Hipotonia esfincteriana & 112 & 31 & - & - \\
\hline Condiloma acuminado & 37 & 22 & 22 & $100,0 \%$ \\
\hline Tumor anal & 3 & 3 & 2 & $66,7 \%$ \\
\hline Total & 1.122 & 729 & $541-31=510$ & $72,8 \%$ \\
\hline
\end{tabular}

deles (Tabela 1 e Figura 1). Fica, destarte, comprometida pelos variados parâmetros utilizados, qualquer tentativa de comparação de nossos achados com os achados dos autores relatados 16, 19, 22, 23 .

Doenças anais concomitantes à $\mathrm{DH}$ e abordagens cirúrgicas das mesmas: é comum a associação de DH com outras doenças anais, merecendo destaques as fissuras, além de outras menos incidentes, como as papilites, as criptites, as fístulas, os prolapsos, os condilomas e os pólipos. Diagnósticos concomitantes ao diagnóstico da $\mathrm{DH}$, por ocasião do exame proctológico, como doenças paralelas ou secundárias, foram feitos em 1.122 pacientes $(12,1 \%$ ) (Tabela 2, 3 e 4 e Figura 2, 3, e 4), destacando-se $541(5,8 \%)$ casos de fissuras anais, seguindo-se $312(3,4 \%)$ casos de hipertrofia de papilas anais, $117(1,3 \%)$ casos de fístula anal, $112(1,2 \%)$ casos de hipotonia anal com incontinência parcial, $37(0,4 \%)$ casos de condilomas anais acuminados e $3(0,03 \%)$ de tumores perianais.

Na série de Ganchrow et al. ${ }^{17}$, dos 2.038 pacientes operados de $\mathrm{DH}$ foi a seguinte a incidência de DAC à DH: 26,9\% (548 casos), dentre os quais fissuras anais ( 344 casos, $16,9 \%$ ), hipertrofia de papilas anais ( 69 casos, $4,0 \%)$, fístulas anais (63 casos, 3,2\%), prurido anal ( 26 casos, $1,3 \%$ ), estenoses anais ( 22 casos, $1,1 \%)$, abscessos anais (13 casos, $0,6 \%)$, condilomas anais ( 6 casos, $0,3 \%)$ e doença pilonidal ( 5 casos, $0,2 \%$ ).

Entre os 2.417 pacientes operados de DH, 729 $(30,1 \%)$ foram operados de outras doenças anais, destacando-se 13,1\% (317 pacientes) de fissura anal, 11,0\% (267 pacientes) de hipertrofia de papilas anais, 3,7\%

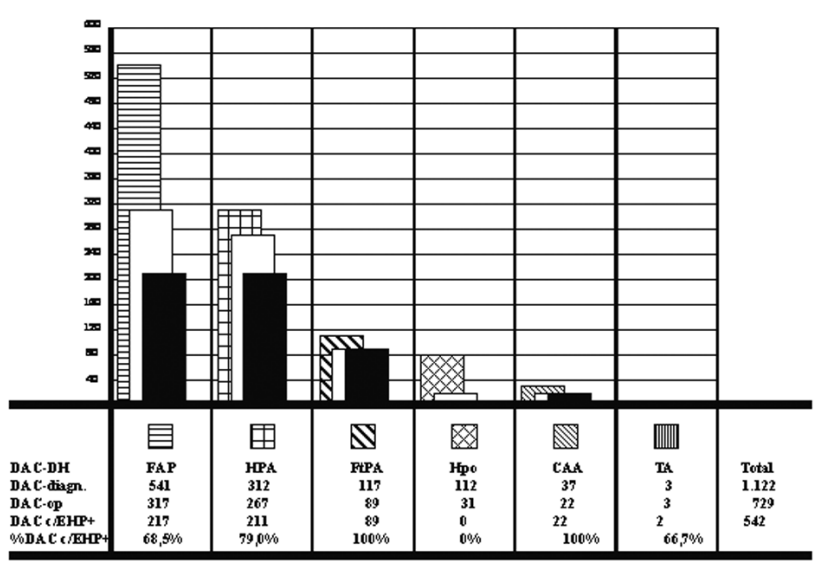

Figura 4 - Incidência de doenças anais concomitantes à doença hemorroidária (DAC-DH) diagnosticadas (DAC-diagn) (colunas quadriculadas); incidência de DAC operadas e de peças enviadas para exame histopatológico (DAC-op) (colunas em xadrez); e incidência, em números absolutos e percentuais, de resultados positivos do EHP para a DAC de suspeita clínica (colunas lisas) (DAC $c / E H P+e \% D A C c / E H P+)$; englobando, respectivamente cada uma das DAC - fissura anal posterior (FAP), hipertrofia de papila anal (HPA), fístula perianal (FtPA), hipotonia esfincteriana (Hpo), condiloma anal acuminado (CAA) e tumor perianal (TPA).

(89 pacientes) de fístula anal, 1,3\% (31 pacientes) de hipotonia anal com incontinência anal parcial, 0,9\% (22 pacientes) de condilomas anais acuminados e $0,1 \%$ (3 pacientes) de tumores anais malignos (Tabela 2,3 e 4 e Figura 2, 3, e 4).

Na série de Ganchrow et al. ${ }^{17}$, dos 2.038 pacientes operados de DH, 955 foram também operados de DAC à DH (46,8\%), assim distribuídas: fissura anal (433 casos, 21,3\%), fistulectomia (120 casos, 5,9\%), papilectomia (50 casos, 2,4\%), drenagem de abscesso 
(21 casos, 1,0\%), ressecção de doença pilonidal (20 casos, $0,9 \%$ ) e anoplastia (22 casos, 1,0\%). Foram feitas, ainda 244 anoplastias (12,0\%), não visando DAC, fato que diminui a incidência global de abordagens cirúrgicas de DAC de $46,8 \%$ para $34,9 \%$.

Comparando nossos achados (Tabela 2, 3 e 4 e Figura 2, 3, e 4) com os de Ganchrow et al. ${ }^{17}$, no tocante às cirurgias de DAC à DH, subtraindo-se as 244 anoplastias, verificamos o seguinte: 2.417 pacientes operados contra 2.038; operados de DAC $30,1 \%$ contra $34,9 \%$; operados de fissura anal $11,0 \%$ contra $21,3 \%$ ); operados de hipertrofia de papilas anais $3,7 \%$ contra $2,4 \%$; operados de fístula anal $1,3 \%$ contra $5,9 \%$; não houve, na série de Ganchrow et al ${ }^{17}$ casos de cirurgias em portadores de hipotonia anal com incontinência anal parcial, de condilomas anais acuminados e de tumores anais malignos; em contrapartida não houve em nossa série pacientes operados de doença pilonidal, estenoses anais e abscessos perianais. Verificamos, portanto, apesar de semelhanças globais (30,1\% contra $34,9 \%$ ), grande discrepância de incidência de cirurgias específicas em DAC e DH entre nossas séries.

\section{CONCLUSÕES}

1. $12,1 \%$ dos portadores de DH tinham DAC (1.122).

2. $65,0 \%$ das DAC foram operadas (729).

3. $26,0 \%$ das DH foram operadas (2.417 em 9.289).

4. $30,2 \%$ dos operados de DH (2.417) foram operados de DAC (729).

5. As DAC mais comuns foram a fissura anal (541 casos, $5,8 \%$ ) e a hipertrofia de papilas anais (312 casos, $3,4 \%)$.

6. $48,2 \%$ das DAC eram fissuras anais e $27,8 \%$ eram hipertrofia de papilas anais.

7. As DAC mais operadas foram a fissura anal (317 casos, 28,3\%) e a hipertrofia de papilas anais (267 casos, $23,8 \%$ ).

8. $100 \%$ dos tumores anais, $85,6 \%$ das hipertrofias de papilas anais e 76,0\% das fístulas anais foram operadas.

9. A confirmação dos diagnósticos das DAC pelo exame histopatológico foi de $72,8 \%$, mais dos condilomas anais e fístulas anais $(100,0 \%)$ e das hipertrofias de papilas anais $(79,0 \%)$.

ABSTRACT: In a 38-year period of practice in Coloproctology, the author had the opportunity to attend 34,000 patients and the diagnosis of hemorrhoid as the main cause of the symptoms presented was achieved in 9,289 patients (27.3\%) being hemorrhoid associated with several anorectal diseases in 1,122 patients (12.1\%). 2,417 patients of the patients underwent hemorrhoidectomy (26.0\%) and 729 of theese patients were operated on for associated anal diseases at the same time (65.0\%). The most frequently diagnosed associated anal diseases were anal fissures (541 cases, $5.8 \%)$ and hyperthrofied anal papila (312 cases, $3.4 \%)$ folowed by anal fistulae ( 117 cases, $1.3 \%$ ), partial fecal incontinence (112 cases, $1.2 \%)$, anal condyloma ( 37 cases, $0,4 \%)$ and anal tumors $(3$ cases, $0,03 \%)$.

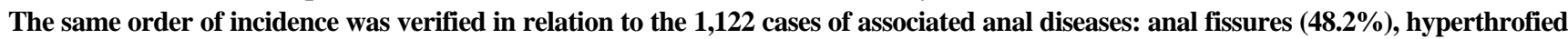
anal papila $(\mathbf{2 7 . 8 \%})$, anal fistulae $(\mathbf{1 0 . 4 \%})$, partial fecal incontinence $(\mathbf{1 0 . 0 \%})$, anal condyloma $(\mathbf{3 . 3} \%)$ and anal tumors $(\mathbf{0 , 3} \%)$. As far as associated anal diseases are concerned (1,122 cases), the incidence of surgery was $65.5 \%$ (729 patients) in this order: fissurectomy (317 cases, $28.3 \%$ ), anal papilectomy (267 cases, $23.8 \%$ ), anal fistulectomy (89 cases, $7.9 \%)$, partial fecal incontinence (31 cases, $2.8 \%)$, resection of anal condylomata ( 22 cases, $1.9 \%)$, resection of anal tumors ( 3 cases, $0.3 \%)$. And as far as each associated anal disease is concerned the incidence of surgery was the following: resection of anal tumors $(\mathbf{1 0 0 , 0 \%})$, papilectomy $(\mathbf{8 5 . 6 \%})$, fistulectomy $(\mathbf{7 6 . 0 \%})$, resection of anal condylomata $\mathbf{5 9 . 6 \%}$ ), fissurectomy (58.6\%) and partial fecal incontinence (25.8\%). Associated anal diseases with the highest proportion of confirmation of proctologic diagnosis by the histopathologic examination were anal fistula (100.0\% of 89 operated patients), anal condyloma (100.0\% of 22 operated patients), hyperthrophied anal papilla (79.0\% - 211 - of 267 operated patients), anal fissure (68.5\% - 217 - of 317 operated patients) and anal cancer (66.7\% - 2 of 3 operated patients).

Key words: Hemorrhoids; Anal diseases.

\section{REFERÊNCIAS}

1. Andrade Filho, J.S. Biópsias do ponto de vista do patologista. In: Fonseca F.P., Savassi, PR. Cirurgia ambulatorial. 2a. ed., Editora Guanabara Koogan, 196-200, 1987.

2. Bogliolo L. Patologia. $5^{\text {a }}$ ed. Rio de Janeiro: Ed. Guanabara Koogan; 1994.
3. Brown DR, Bryan JT, Cramer H, Katz BP, Handy V, Fife KH. Detection of multiple human Papillomavirus types in condylomata acuminata from immunosuppressed patients. J Infect Dis, 1994; 170(4):759-65.

4. Carneiro; LLR, Zerbini JC, Cruz GMG, Costa LMP, Silva IG, Miranda SML, Teixeira RG, Minarrini J, Lanna D, Júnior HNV, Lima MJR, Luz MMP, Pereira FSA, Silva GM, Khouri 
RM, Oliveira KA e Marques ET). Exame histopatológico de peças cirúrgicas de hemorroidectomia. Rev Bras de Coloproctologia, 19 - Suplemento 1, página 43, (1999).

5. Chu QD, Vezeridis MP, Libbey NP, Wanebo HJ. Giant Condyloma Acuminatum (Buschke-Löwenstein Tumor) of the Anorectal and Perianal Regions. Dis Colon Rectum, 1994; 37:950-7.

6. Congilosi SM, Madoff RD. Current Therapy for recurrent and Extensive Anal Warts. Dis Colon Rectum, 1995; 38:1101-7.

7. Coper, P.H., Mills S.E. Allen, M.S. Malignant melanoma of the anus: Report of 12 patients and analysis of 255 adicional cases. Dis. Colon Rectum, 25: 693, 1982.

8. Cruz GMG, Oliveira AT et Rodrigues JVL. Estudo retrospectivo de 870 exames proctológicos em pacientes de área metropolitana. Rev Bras de Coloproctologia, 16 (4), 200-205, 1996.

9. Cruz GMA, Oliveira AT, Rodrigues JVL. Manifestações coloproctológicas em 42 pacientes portadores de síndrome de imunodeficiência adquirida (AIDS). Rev Bras de Coloproctologia, 17 (2), 105-116, 1997.

10. Cruz GMG, Silva IG, Teixeira RG, Andrade Filho JS, Pena GPM. Melanoma retal: apresentação de três casos e revisão da literatura. Rev Bras de Coloproctologia, 18 (3), 202-207, 1998.

11. Cruz GMG. Tumores Malignos do Anus e Canal Anal. In: Castro LP, Rocha PRS, Carvalho DGT. Tópicos em Gastroenterologia 3. Ed. MEDSI, Rio de Janeiro, 1992.

12. Cruz GMG. Papilites e Cripitites Anais. In: Cruz GMG. Livro Texto "Coloproctologia", Volume II - "Coloproctologia Propedêutica Nosológica”, Editora Revinter, Rio de Janeiro, RJ, 1999. Vol II, parte VII, cap. 80, 1183 - 1186.

13. Cruz GMG. Tumores malignsos do Ânus e Canal Anal. In: Cruz GMG. Livro Texto "Coloproctologia", Volume II "Coloproctologia - Propedêutica Nosológica”, Editora Revinter, Rio de Janeiro, RJ, 1999. Vol II, parte VII, cap. 81, 1187 - 1200.

14. Cunha AS. Parasitoses Intestinais. In: Dani R \& Paula Castro L, Gastroenterologia Clínica. Ed. Guanabara $3^{a}$ edição, Ed. Guanabara, Rio de Janeiro, 1995.

15. Fillmann EEP, Fillmann LS. Fístulas Anorretoperineais. In: Cruz GMG. Livro Texto "Coloproctologia", Volume II "Coloproctologia - Propedêutica Nosológica”, Editora Revinter, Rio de Janeiro, RJ, 1999. Vol II, parte VII, cap. 78, 1171 - 1176.

16. Fry, G.A. Martin, W.J., Dearing, W.H. Culp, C.E. Primary actinomycosis of the rectum with multiple perianal and perineal fistulae. Mayo Clinic Proc., 40: 296-259, 1985.

17. Ganchrow MI, Mazier WP, Friend WG et a.1. Hemorrhoidectomy revisited - a computer analysis of 2.038 cases. Dis Colon Rectum 1971; 14: 128-133.

18. Habr-Gama A \& Bocchini SF. Criptites e Papilites. In: Pinotti HW. Tratado de Clínica Cirúrgica do Aparelho Digestivo. ATHENEU, São Paulo, 1994; cap.133:1102-03.

19. Haas P. A. and cols. - The pathogenesis of Hemorrhoids. Diseases of the Colon and Rectum, 1984 July; vol 27 (7): 442-50.
20. Iglésias, JDF. Aspectos Médicos das Parasitoses Humanas. 1aㅗ ed. Rio de Janeiro: Ed. MEDSI, 1996.

21. Iglésias JDF. Exames Laboratoriais das Doenças Infectocontagiosas e Parasiárias Anorretais. In: Cruz GMG. Livro Texto "Coloproctologia”, Volume I - "Coloproctologia - Propedêutica Geral”, Editora Revinter, Rio de Janeiro, RJ, 1998. Vol I, parte III, cap. 23, 258-274.

22. Iwagaki H, Higuchi Y, Fuchimoto S \& Orita K. The laser treatment of hemorrhoids: results of a study on 1816 patients. Jpn J Surg 1989; 19: 658-61.

23. Johanson JF, Rimm A. Optimal nonsurgical treatment of hemorrhoids: a comparative analysis of Infrared coagulation, Rubber Band ligation and Injection sclerotherapy. Am J Gastroenterol, 1992; 87: 1600-1606.

24. Nahas P, Teixeira MG. Doença de Crohn Perineal. In: Cruz GMG. Livro Texto "Coloproctologia", Volume II "Coloproctologia - Propedêutica Nosológica", Editora Revinter, Rio de Janeiro, RJ, 1999. Vol II, parte VII, cap. 84, 1214 - 1216.

25. Neves DP. Parasitologia humana. $9^{a}$ ed. Rio de Janeiro: Ed. Atheneu; 1995.

26. Nunes A. Exame anatomo-patológico das afecções do ânus e canal anal. In: Cruz GMG. Livro Texto "Coloproctologia", Volume I - "Coloproctologia - Propedêutica Geral”, Editora Revinter, Rio de Janeiro, RJ, 1998. Vol, parte III, cap. 22, 255257.

27. Parks AG. The surgical treatment of haemorrhoids. Br J Surg 1956; 43: 337-351.

28. Pinto JM, Silva AP. In: Cruz GMG. Livro Texto "Coloproctologia", Volume I - "Coloproctologia Propedêutica Geral”, Editora Revinter, Rio de Janeiro, RJ, 1998. Vol I, parte VI, cap. 43, $602-611$.

29. Rosai, J. Ackerman's Surgical Pathology. $8^{\text {th }}$ Ed. Mosby, Cap. 11. 800-814, 1995.

30. Santos HA. Doença Hemorroidária. In: Cruz GMG. Livro Texto "Coloproctologia", Volume II - "Coloproctologia Propedêutica Nosológica”, Editora Revinter, Rio de Janeiro, RJ, 1999. Vol II, parte VIII, cap. 75, 1139 - 1156.

31. Shidham VB. A rapid, economical, and simple method for concentration of Schistosoma mansoni ova in feces. Am J Clin Pathol, 1991; 95:91-5.

32. Singh,R. Nime, R. Mittelman, A. Malignant epithelial tumors of the anal canal. Cancer, 48: 411,1981.

33. Stevens, A. \& Lowe, J. Patologia. 1a. ed. Editora Manole, 513-519, 1996.

34. Wexner SD. Sexually transmitted diseases of the colon, rectum and anus. Dis Colon Retum, 1990;1048-62.

Endereço para correspondência: GERALDO MAGELA GOMES DA CRUZ

Rua Rio de Janeiro, 2017 / 1401

30160-042 - Belo Horizonte - Minas Gerais

Tel.: (31) 3222-8289 